\title{
More risk factors generate lower mortality - a useful advice for improved health in the world
}

\author{
Ginter $\mathrm{E}^{1}$, Simko $\mathrm{V}^{2}$
}

Institute of Preventive and Clinical Medicine, Bratislava, Slovakia. ginter.emil@mail.t-com.sk

The incidence of cardiovascular disease (CVD) is increasing rapidly in countries with low and middle income. That part of the world accounts for an estimated $80 \%$ of the global CVD burden. Even worse, CVD in less affluent countries starts to affect people at younger age. Paradoxically, the CVD risk factors in poor countries are less prominent than in high-income countries. This paradox between low incidence of CVD risk on the one hand and increasing CVD mortality on the other was subjected to critical review of many investigators. In seventeen countries and five continents, they assessed the risk and mortality in more than 156,000 individuals at an average age of fifty, using the INTERHEART Risk Score. Participants were followed for the incidence of cardiovascular disease and death for a mean of 4.1 years (1).

Another INTERHEART study (2) was focused on CVD risk factors. This was a large standardized case-control study involving cases of first myocardial infarction from 52 different countries, compared with their sex- and age-matched controls. Nine modi-

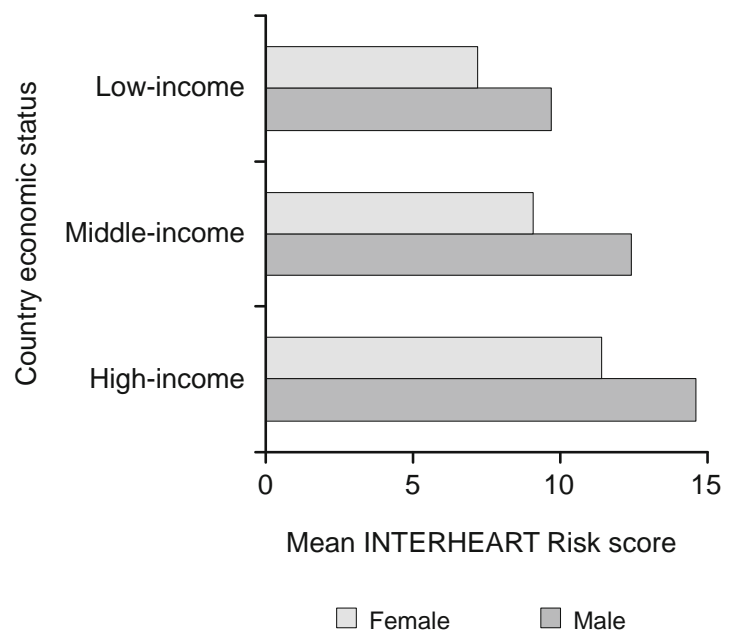

Fig. 1. Country economic status and cardiovascular risk factor burden. Data from Yusuf et al (1).

${ }^{1}$ Institute of Preventive and Clinical Medicine, Bratislava, Slovakia (emeritus), and ${ }^{2}$ State University of New York, Downstate Medical Center at Brooklyn, USA

Address for correspondence: E. Ginter, RND, DSc, Racianska 17, SK83102 Bratislava, Slovakia

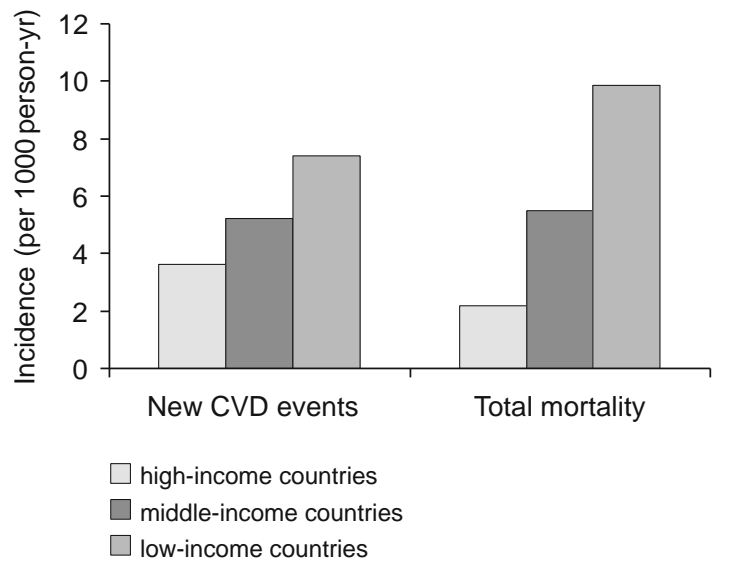

Fig. 2. Country economic status and cardiovascular events. Data from Yusuf et al (1).

fiable risk factors were found to have a globally consistent association with myocardial infarction, namely apolipoprotein levels, smoking, hypertension, diabetes, abdominal obesity, psychosocial factors, adverse dietary factors, insufficient physical exercise, and alcohol consumption.

After having these CVD risk factors specified, their analysis in 17 countries with low, middle, and high-income revealed (1) that the risk declined from affluent to impoverished countries both in urban and rural areas. Conversely, the prevalence of major cardiovascular diseases (myocardial infarction, stroke, and heart failure), as well as total mortality proved to have an opposite trend, namely it increased toward poor countries (Figs 1 and 2).

How can this seeming paradox of high CVD risk and low CVD disorders and related mortality be explained?

Regarding the risks, wealth promotes consumption of calorically rich diet, physical inactivity, and body overweight with adverse health consequences. Still, populations benefiting from this horn of plenty live longer and are afflicted with less CVD than their less fortunate contemporaries in countries with less efficient economy, lower domestic product and more restrictive society. Despite less prominent CVD risks, poor countries have more CVD disorders and mortality associated with shorter life expectancy.

A unifying explanation of this paradox between CVD risks and CVD mortality is the benefit of well-functioning economy and a 


\section{$531-532$}

stable social system. This results in a better control of risk factors (e.g. smoking and alcoholism) and more frequent use of proven pharmacologic therapies and cardiologic revascularization. The success of cardiology in providing evidence-based cost-effective treatments has been a beneficial driving force.

A good example is Germany where reunification added 6.2 years to the life of men in the former communist-dominated East, and 4.2 years to their female counterparts. This phenomenon can be explained by improvements in medical treatment and improved standard of living since reunification. Well-established preventive health care, accessibility of the most up-to-date diagnostic procedures and new revolutionary medications in wealthy countries provide a counterbalance to the increased risk that comes with affluence. Understanding the CVD mortality trends highlights the crucial role of tobacco, unhealthy diet, alcohol, and inactivity as key drivers (4).

Approximately half the decline in rich countries' deaths from CVD may be attributable to reductions in major risk factors and approximately half to evidence-based medical therapies (e.g statins) (5). Advances in contemporary medicine explain another seeming paradox, namely that obesity in the USA as a risk factor is on the rise while CVD mortality is declining.

In fact, epidemiological revelation of the paradox between CVD risk and mortality provides an important encouraging stimulus to improve health in the world. The advice is obvious and practical.

Firstly, in poorer parts of the world, economic stability and social order should be promoted. This is even more pressing now when various armed conflicts contribute to economic devastation and civil disorder.

Secondly, as a society grows more affluent it has to implement measures for lowering and eradication of risk factors even more strongly. Proactive public health measures focused on "upstream" populations are obvious and include preventive medicine, regular control of blood pressure and serum lipids, healthy life style with adequate physical activity and maintenance of balance in energy intake and expenditure, as well as limited smoking and alcohol consumption generating free radicals.

\section{References}

1. Yusuf S, Rangarajan S, Teo $\mathbf{K}$ et al. Cardiovascular risk and events in 17 low-, middle-, and high-income countries. N Engl J Med 2014; 371: 818-827.

2. Yusuf S, Hawken S, Ounpuu S et al. Effect of potentially modifiable risk factors associated with myocardial infarction in 52 countries (the INTERHEART study): case-control study. Lancet 2004; 364: 937-952.

3. European Health for All Database. Copenhagen, WHO Regional Office for Europe, updated July 2013.

4. O'Flaherty M, Buchan I, Capewell S. Contributions of treatment and lifestyle to declining CVD mortality: why have CVD mortality rates declined so much since the 1960s. Heart 2013; 99: 159-162.

5. Ford ES, Ajani UA, Croft JB, et al. Explaining the decrease in U.S. deaths from coronary disease, 1980-2000. N Engl J Med 2007; 356: 2388-2398.

Received October 5, 2014. Accepted January 10, 2015. 